\title{
Fast Convergence Control Approach for High Dimensional Chaotic Systems
}

\author{
Hsun-Heng Tsai \\ Department of Mechanical Engineering, National Central University. \\ Chyun-Chau Fuh \\ Department of Mechanical and Marine Engineering, National Taiwan Ocean University. \\ Pi-Cheng Tung \\ Department of Mechanical Engineering, National Central University, Chung-Li, Taiwan 32054, ROC., \\ t331166@ncu.edu.tw
}

Follow this and additional works at: https://jmstt.ntou.edu.tw/journal

8 Part of the Mechanical Engineering Commons

\section{Recommended Citation}

Tsai, Hsun-Heng; Fuh, Chyun-Chau; and Tung, Pi-Cheng (2002) "Fast Convergence Control Approach for High Dimensional Chaotic Systems," Journal of Marine Science and Technology. Vol. 10: Iss. 2, Article 1.

DOI: $10.51400 / 2709-6998.2304$

Available at: https://jmstt.ntou.edu.tw/journal/vol10/iss2/1

This Research Article is brought to you for free and open access by Journal of Marine Science and Technology. It has been accepted for inclusion in Journal of Marine Science and Technology by an authorized editor of Journal of Marine Science and Technology. 


\title{
FAST CONVERGENCE CONTROL APPROACH FOR HIGH DIMENSIONAL CHAOTIC SYSTEMS
}

\author{
Hsun-Heng Tsai*, Chyun-Chau Fuh**, and Pi-Cheng Tung***
}

Key words: chaos, control, Jacobian linearization, pole placement.

\begin{abstract}
A simple and efficient method for controlling high dimensional discrete-time chaotic systems is proposed in this paper. This method is implemented similar to the OGY method, and is feasible for practical experiments. The key component is to assign the eigenvalues of a linearized map by using the well-known pole placement technique. According to the Cayley-Hamilton theorem, the trajectory will converge to the desired fixed point after $n$ iterations at most ( $n$ is the dimension of the map), if the real trajectory of the chaotic system falls within the neighborhood of the desired fixed point. The proposed approach improves the convergence rate and the robustness of the OGY method, especially for the case where the modulus of the stable eigenvalue is close to unity. The simulations illustrate the performance of our presented controller for controlling a chaotic system compared to the OGY method.
\end{abstract}

\section{INTRODUCTION}

Chaotic behavior often occurs in engineering's and natural systems [1-6]. In the past it has been seen as irregular or unpredictable and was often attributed to random external influences. Further studies have shown that chaotic behavior is deterministic, and is a typical characteristic of nonlinear systems. However, in many engineering applications, chaos is seen as an undesirable phenomenon, because chaotic motion can cause performance degradation and restrict the operating range of dynamic systems. Therefore, developing strategies to control chaos, based on the features of chaotic motion, is necessary. The famous Ott-Grebogi-York (OGY) paper [7], that pointed out the possibility of chaos control, was the seed for a large body of research that is still rapidly growing.

Paper Submitted 12/04/01, Accepted 02/04/02. Author for Correspondence: Pi-Cheng Tung.

*Department of Mechanical Engineering, National Central University.

**Department of Mechanical and Marine Engineering, National Taiwan Ocean University.

***Department of Mechanical Engineering, National Central University, Chung-Li, Taiwan 32054, ROC. E-mail: t331166@ncu.edu.tw
The OGY method is based on Jacobian linearization. First, one chooses an appropriate unstable periodic orbit embedded in a strange attractor. The linearized model is then estimated and a small region around the desired periodic orbit is defined. The main feature of the OGY controller is that assigns the unstable eigenvalue of the linearized model to zero, but does not move the stable eigenvalue. Hence, when a trajectory falls into the defined region, small parameter perturbations are applied to force the trajectory into the stable manifold. The trajectory will then approach the desired periodic orbit asymptotically. Unfortunately, for higher-dimensional chaotic systems, especially for a chaotic attractor with two or more positive Lyapunov exponents, the original OGY method fails. In order to lesson the disadvantages of the original OGY method, many modified methods and other approaches, which can be applied to high dimensional chaotic systems, have successively been proposed [8-12]. However, no general method of control exists which is adaptable to all chaotic systems due to their diversity.

The solution to the problem of determining the feedback gain, so that the eigenvalues of the controlled system have specified values, has been extensively studied, the so-called pole-placement technique. The position of the specified eigenvalues will determine the performance of the controlled system, so how to locate controlled system eigenvalues becomes an important issue. Modified OGY control schemes, such as a control law based on pole-placement techniques [13], a scheme for implementation of the convergence rate [14], and the optimization of control [15] have been proposed. Nevertheless, in these papers the assigned eigenvalues only confirm the asymptotical stability of the system. Here, we present a simple and efficient method for controlling discrete-time chaotic systems based on the OGY method and the linear feedback theory. In the OGY method, if the modulus of the stable eigenvalue is close to unity, the tracking error convergence will be very slow, because the orbit will approach the desired periodic orbit only at the geometri- 
cal rate of the stable eigenvalue. However, the key to the proposed method is to assign all of the eigenvalues of a linearized map to zero, by applying the well-known pole placement technique [16] and Cayley-Hamilton's theorem, which can control an $n$-dimensional chaotic system into stable region in the desired $n$ steps. Since this method is also based on a linear approximation of the chaotic map, it is feasible in practical experiments similar to the OGY method. Whenever the trajectories fall in the neighborhood of the desired fixed point, our proposed method requires less effort to control, and has a faster convergence rate, to stabilize a chaotic system, than does the OGY method.

\section{CRITERIA FOR CONTROL}

In practical experiments the exact nonlinear dynamic equations of a chaotic system are usually unknown. Sometimes the only measurable signal may be a scalar output signal, for instance $y(t)$, in which case, one can reconstruct the chaotic attractor using the embedding technique [17]. Since continuous dynamic systems can be regarded as discrete maps on the Poincare surface, only discrete-time dynamic systems are considered here. A delay-coordinate vector in the $n$-dimensional embedding space is defined as follows:

$$
\mathbf{y}(k)=\left[\begin{array}{lllll}
y(k) & y(k-1) & y(k-2) & \ldots & y(k-(n-1))
\end{array}\right]^{T},
$$

where $k$ denotes the sampling instant and is an integer ranging from $-\infty$ to $+\infty$. The bold capitals and bold lowercase letters denote the matrices and vectors, respectively. We let $\bar{y}_{i}, i=1, \ldots, N$, be an unstable periodic $-N$ orbit embedded in the strange attractor. If we suppose the performance of the periodic- $N$ orbit satisfies our requirements. Then by using the delaycoordinate vector, the desired periodic- $N$ orbit can be represented as follows:

$$
\begin{aligned}
& \overline{\mathbf{y}}(k)=\left[\begin{array}{llll}
\bar{y}_{i+n-1} & \bar{y}_{i+n-2} & \cdots & \bar{y}_{i}
\end{array}\right]^{T}, \\
& i=\bmod (k, N)+1 .
\end{aligned}
$$

The tracking error can be defined as:

$$
\begin{aligned}
\mathbf{e}(k)= & \mathbf{y}(k)-\overline{\mathbf{y}}(k) \\
= & {\left[y(k)-\bar{y}_{i+n-1} y(k-1)-\bar{y}_{i+n-2}\right.} \\
& \left.\cdots y(k-n+1)-\bar{y}_{i}\right]^{T} .
\end{aligned}
$$

In additionally, we assume that $p$ is an accessible parameter that can be externally perturbed. The constant $\bar{p}$ denotes the nominal value. The small parameter perturbation about $\bar{p}$ is defined as $u(k)=p(k)-\bar{p}$. The error dynamics can then be described using nonlinear difference equations of the form:

$$
\mathbf{e}(k+1)=\mathbf{f}(\mathbf{e}(k), u(k)),
$$

where $\mathbf{f}$ is assumed to be continuously differentiable, at least locally in the neighborhood of the fixed point $(\mathbf{e}, u)$ $=(\mathbf{0}, 0)$. A linear approximation of the above system near the fixed point is given by:

$$
\mathbf{e}(k+1)=\mathbf{A e}(k)+\mathbf{b} u(k),
$$

where

$$
\mathbf{A}=\left.\frac{\partial \mathbf{f}}{\partial \mathbf{e}}\right|_{\substack{\mathbf{e}=0 \\ u=0}}, \quad \mathbf{b}=\left.\frac{\partial \mathbf{f}}{\partial \mathbf{e}}\right|_{\substack{\mathbf{e}=0 \\ u=0}} .
$$

Let us briefly recall the OGY control method. Here we restrict ourselves to a two-dimensional system (i.e., $n=2$ ). Let $\lambda_{s}$ and $\lambda_{u}$ be the stable and unstable eigenvalues of the Jacobian A, respectively. The directions of the stable and unstable manifolds of the equilibrium are given by $\mathbf{e}_{s}$ and $\mathbf{e}_{u}$, respectively. If $\mathbf{f}_{s}$ and $\mathbf{f}_{u}$ are the contravariant basis vectors defined by $\mathbf{f}_{s}^{T} \mathbf{e}_{s}=\mathbf{f}_{u}^{T} \mathbf{e}_{u}=1$, $\mathbf{f}_{s}^{T} \mathbf{e}_{u}=\mathbf{f}_{u}^{T} \mathbf{e}_{s}=0$, then the OGY control law is as follows [4]:

$$
u(k)=\frac{\lambda_{u} \mathbf{f}_{u}^{T}}{\left(\lambda_{u}-1\right) \mathbf{f}_{u}^{T} \mathbf{w}} \mathbf{e}(k) \equiv \mathbf{k e}(k),
$$

where $\mathbf{w}=(\mathbf{I}-\mathbf{A})^{-1} \mathbf{b}$ and it is assumed that $\mathbf{f}_{u}^{T} \mathbf{w} \neq 0$ (or equivalently $\left.\mathbf{f}_{u}^{T} \mathbf{b} \neq 0\right)$. This is equivalent to the simpler formula [18]:

$$
u(k)=\frac{-\lambda_{u} \mathbf{f}_{u}^{T}}{\mathbf{f}_{u}^{T} \mathbf{b}} \mathbf{e}(k)=\mathbf{k e}(k) .
$$

The main feature of the OGY method is to assign the unstable eigenvalue of the linearized model to zero, but not to move the stable eigenvalue. The original OGY method may have some disadvantages. First, if the modulus of the stable eigenvalue $\lambda_{s}$ is close to unity, the convergence of the tracking error is very slow, because the orbit will approach the fixed point at a geometrical rate $\lambda_{s}$. In addition, the controlled system is less robust in term of disturbance and noise if the modulus of $\lambda_{s}$ is close to unity. Moreover, for a higherdimensional chaotic system, especially for a chaotic attractor with two or more positive Lyapunov exponents, the original OGY method fails.

In fact, if $(\mathbf{A}, \mathbf{b})$ is controllable, i.e., the controllability matrix:

$$
\mathbf{U}=\left[\begin{array}{llll}
\mathbf{b} & \mathbf{A b} & \ldots & \mathbf{A}^{n-1} \mathbf{b}
\end{array}\right],
$$


has the rank $n$. Then by using linear feedback in the form $u(k)=\mathbf{k e}(k)$, the eigenvalues of $\mathbf{A}+\mathbf{b k}$ can be arbitrarily assigned, provided the complex conjugate eigenvalues appear in pairs. This implies that we can choose an arbitrary feedback gain $\mathbf{k}$, such that all of the eigenvalues of $\mathbf{A}+\mathbf{b k}$ lie inside the unit circle to stabilize the equilibrium of equation (5).

In this paper, a chaos control technique based on the OGY method and linear feedback theory is presented. The kernel of the proposed method is to assign all of the eigenvalues to zero using the well-known pole placement technique. According to conventional digital control theory, assigning all of the eigenvalues of a linear discrete-time system to zero implies that one has to design a high-gain controller. Using such controllers in linear systems can cause two problems. The first is the saturation problem of the real actuator/amplifier. The second is that a high-gain controller usually results in self-excited oscillation due to the exciting of the uncertain high-frequency mode of the system. Therefore, assigning the eigenvalues to zero is not generally allowed in conventional linear control method.

Nevertheless, most techniques developed for controlling chaotic systems are based on linearized models, such as that in equation (5). These controllers are available only when the real trajectory of the chaotic system falls in the neighborhood of a desired fixed point (i.e., the Euclidean norm of the error vector $\|\mathbf{e}(k)\|$ is close to zero). The small error vector $\mathbf{e}(k)$ is multiplied by a finite gain $\mathbf{k}$ and the product $u$ is sure to be small enough. This implies that, with a limited control input, the use of the so-called high-gain controllers in chaotic systems is feasible. In addition, using a high-gain controller in a chaotic system will not produce the disadvantages induced in linear systems. Since we can disable the controller if the actual trajectory in a chaotic system is far from the desired fixed point or the control input exceeds the preset range. Although the trajectory departs temporarily from the desired fixed point after the controller is disabled, this uncontrolled trajectory will once more fall in the neighborhood of the desired fixed point after some iterations.

In this paper, only one class of chaotic systems, those controllable at least locally in the neighborhood of the desired fixed point, are considered. We first represent the linearized model of a controllable chaotic system near the fixed point by the form in equation (5). According to the pole placement technique, the state feedback gain for assigning all of the eigenvalues to zero is then given by:

$$
\mathbf{k}=\left[\begin{array}{llll}
a_{n} & a_{n-1} & \ldots & a_{1}
\end{array}\right](\mathbf{U W})^{-1},
$$

where the controllability matrix $\mathbf{U}$ is defined as equa- tion (9), and:

$$
\mathbf{W}=\left[\begin{array}{ccccc}
a_{n-1} & a_{n-2} & \cdots & a_{1} & 1 \\
a_{n-2} & a_{n-3} & \cdots & 1 & 0 \\
\vdots & \vdots & \ddots & \vdots & \vdots \\
a_{1} & 1 & \cdots & 0 & 0 \\
1 & 0 & \cdots & 0 & 0
\end{array}\right]
$$

We let $\mathbf{u}(k)=\mathbf{K e}(k)$. Thus equation (5) becomes:

$$
\mathbf{e}(k+1)=(\mathbf{A}+\mathbf{b k}) \mathbf{e}(k),
$$

where the resulting matrix $\overline{\mathbf{A}}=\mathbf{A}+\mathbf{b k}$ has an eigenvalue of 0 with multiplicity $n$. Since the characteristic polynomial of $\overline{\mathbf{A}}$ is:

$$
\Delta(\lambda)=\lambda^{n},
$$

and

$$
\mathbf{e}(k+r)=\overline{\mathbf{A}}^{r} \mathbf{e}(k),
$$

the Cayley-Hamilton theorem implies:

$$
\overline{\mathbf{A}}^{r}=0, \quad \forall r \geq n .
$$

Hence, the trajectory will be determined and will converge to the desired fixed point after $n$ at most iterations, if the real trajectory of the chaotic system falls in the neighborhood of the desired fixed point. This is different from the OGY method, where the convergence of the tracking error may be very slow, because the orbit will approach the fixed point at the geometrical rate $\lambda_{s}$.

\section{NUMERICAL EXAMPLE}

Consider a two-dimensional nonlinear map described as follows:

$$
\begin{aligned}
& x_{1}(k+1)=p\left(x_{1}(k)-x_{1}^{2}(k)\right), \\
& x_{2}(k+1)=(1-q) x_{1}(k)+q x_{2}(k),
\end{aligned}
$$

where $p=p_{0}+u(k)$, and $u(k)$ is the control input. The corresponding parameters are $p_{0}=4$ and $q=0.5$. With such parameters, an uncontrolled system exhibits a chaotic attractor. A dense set of unstable periodic orbits embedded within a strange attractor is the main characteristic of a chaotic system. Without loss of generality, let the period-1 orbit $\overline{\mathbf{x}}=\left[\begin{array}{ll}\bar{x}_{1} & \bar{x}_{2}\end{array}\right]^{T}=\left[\begin{array}{ll}0.75 & 0.75\end{array}\right]^{T}$, embedded within the strange attractor, be the desired fixed point. For simplicity, assume that the exact nonlinear dynamic equation of the chaotic system is 
unknown, but the state vector $\mathbf{x}(k)=\left[\begin{array}{ll}x_{1}(k) & x_{2}(k)\end{array}\right]^{T}$, is measurable. Define the tracking error, $\mathbf{e}(k)=\mathbf{x}(k)-\overline{\mathbf{x}}$. The linear approximation of the chaotic system near the fixed point can be estimated as follows:

$$
\begin{aligned}
\mathbf{e}(k+1) & =\mathbf{A e}(k)+\mathbf{b u}(k) \\
& =\left[\begin{array}{cc}
-2 & 0 \\
0.5 & 0.5
\end{array}\right] \mathbf{e}(k)+\left[\begin{array}{c}
0.1875 \\
0
\end{array}\right] u(k) .
\end{aligned}
$$

We emphasize that the desired fixed point $\overline{\mathbf{x}}$, and the parameter matrices $\mathbf{A}$ and $\mathbf{b}$, are all experimentally accessible. Using the pole placement technique, we get:

$$
u(k)=\mathbf{k e}(k)=\left[\begin{array}{ll}
8 & -2.6667
\end{array}\right] \mathbf{e}(k) .
$$

The feedback gain will shift the eigenvalues in the linear error model from $\{-2,0.5\}$ to $\{0,0\}$. If the OGY method is applied, the controller can be determined as follows

$$
u(k)=\mathbf{k e}(k)=\left[\begin{array}{ll}
10.6667 & 0
\end{array}\right] \mathbf{e}(k) .
$$

This feedback gain assigns the eigenvalues in the linear error model to be $\{0,0.5\}$. The results are shown in Figure 1, where the initial conditions are $\mathbf{e}_{0}=[0$. $002590.00966]^{T}$ and $\Omega_{0}=\left\{\mathbf{e} \in \mathbf{R}^{2}:\|\mathbf{e}\|<3.5 \times 10^{-4}\right\}$. Using our proposed method, the trajectory $\mathbf{e}$, originating from $\mathbf{e}_{0}$, tends to become $\Omega_{0}$ after only two iterations. If we use the OGY method, at least five iterations are required to convert trajectory $\mathbf{e}$ into $\Omega_{0}$ from $\mathbf{e}_{0}$. Note that the nonlinear terms of the exact system and the truncation error may cause a small deviation.

Our proposed method is also able to stabilize highperiodic orbits. The controlled result is shown in Figure 2, where we successively stabilize period-5, period-2, period-3, and period- 4 orbits. In this example, the control input is allowed to vary in the range of $|u(k)| \leq$ 0.2 , which is about $5 \%$ of the nominal value of $p_{0}=4$.

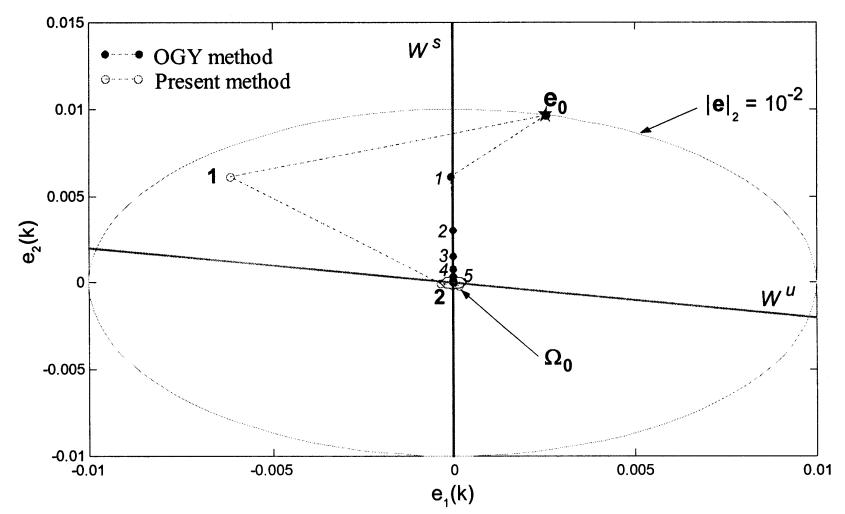

Fig. 1. Comparison of the trajectories originating from $\mathbf{e}_{0}$ tending to region $\Omega_{0}$ with the OGY method $(\bullet . . \bullet)$ and the present method $(\circ \ldots \circ)$.
From $k=1$ to 10000 , the proposed method is applied to stabilize period-5, from 10001 to 20000 to stabilize period-2, from 20001 to 30000 to stabilize period-3, and from 10001 to 20000 to stabilize period-4.

In the following, we will compare the performance of our method with the OGY method. Let us first define $\Omega=\left\{\mathbf{e} \in \mathbf{R}^{2}:\|\mathbf{e}\|<10^{-6}\right\}$ and $\mathbf{C}_{r}=\left\{\mathbf{e} \in \mathbf{R}^{2}:\|\mathbf{e}\|=\right.$ $\left.10^{-6} r\right\}$, where $r$ is a parameter greater than one. Thus, we can compute the average number of iterations $\langle\kappa\rangle$ required to reach $\Omega$ from 200 randomly selected initial conditions on $\mathbf{C}_{r}$. Figure 3 shows the variation in $\langle\kappa\rangle$ as a function of $r$. Using our proposed method, the value
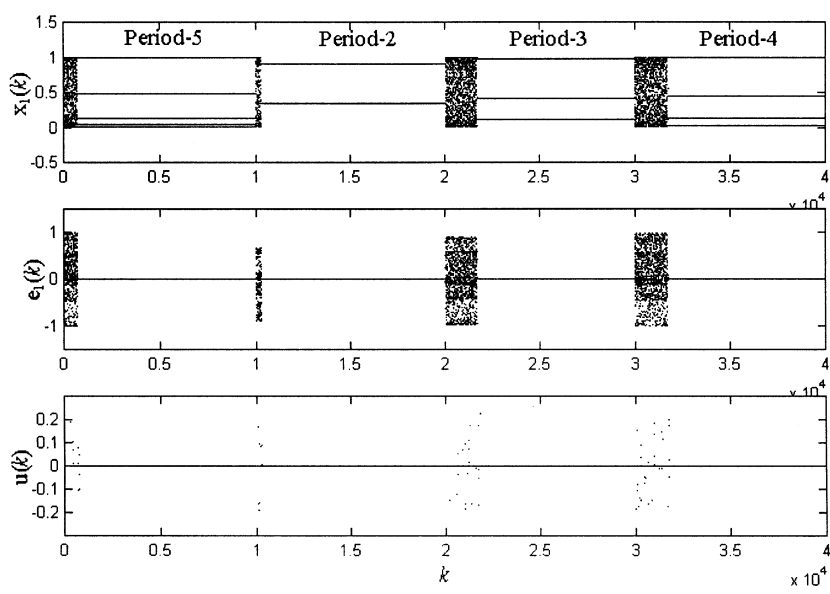

Fig. 2. Transition between periodic orbits from $\mathrm{P} 5 \rightarrow \mathrm{P} 2 \rightarrow \mathrm{P} 3 \rightarrow \mathrm{P} 4$, where the control input is allowed to vary in the range of $|u(k)|<0.2$. From $k=1 \sim 10000$ the proposed method is applied to stabilize period-5, from to stabilize period-2, from 30001 40000 to stabilize period-3, from to stabilize period-4. (a) Time response of $x_{1}(k)$; (b) Tracking error $e_{1}(k)=x_{1}(k)-\bar{x}_{1}$; (c) Control input $u(k)$.

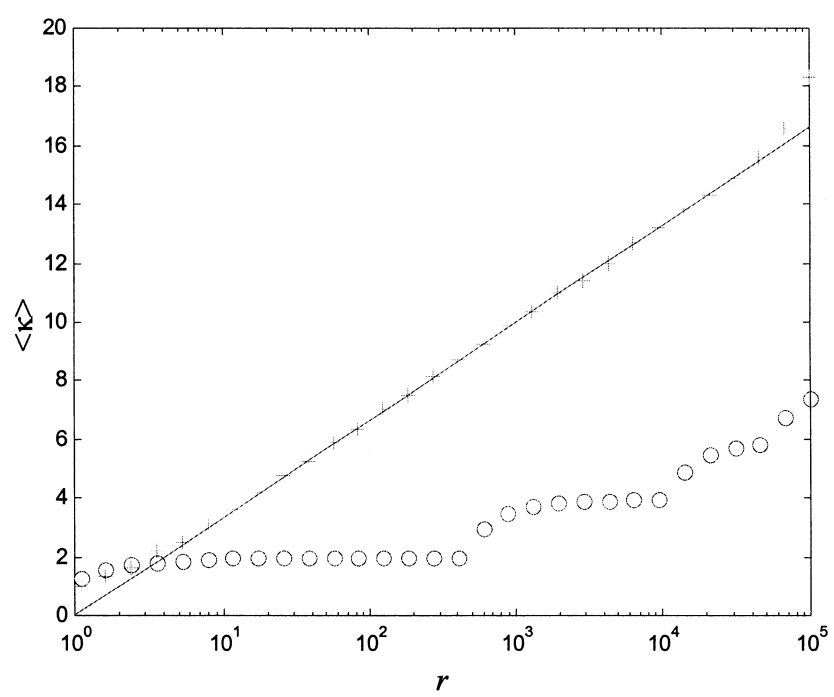

Fig. 3. Average number of iterations $\langle\kappa\rangle$ required to reach $\Omega$ from 200 randomly selected initial conditions on $\mathbf{C}_{r}$ with the OGY method $(\times)$ and the present method (o). 
of $\langle\kappa\rangle$ is approximately 2 as $r$ increases from 2 to 400 . This result is consistent with our previous account.

When $r$ is greater than 400 , the value of $\langle\kappa\rangle$ increases gradually due to the nonlinear terms. Therefore, we can conclude that linear equation (17) is a good approximation of this nonlinear system in the region where $\Omega_{L}=\left\{\mathbf{e} \in \mathbf{R}^{2}:\|\mathbf{e}\|<4 \times 10^{-4}\right.$. If we use the OGY method, Figure 3 shows that the average number of iterations $\langle\kappa\rangle$ increases with increasing $r$ and following a logarithmic relationship:

$$
\langle\kappa\rangle \sim \log _{\lambda_{s}}\left(\frac{1}{r}\right),
$$

or

$$
\lambda_{s}^{\langle\kappa\rangle} \sim \frac{1}{r}
$$

where $\lambda_{s}=0.5$. This result verifies the previous statement, that the orbit will approach the fixed point at the geometrical rate $\lambda_{s}$. Therefore, the OGY method is unsuitable for cases in which the modulus of the stable eigenvalue $\lambda_{s}$ is close to unity, because the convergence of the tracking error will be very slow, as shown in Figure 4.

In Additional, in order to verify that our proposed method does not require larger parameter perturbations, the maximum input $u_{\text {max }}$, required to reach $\Omega$ from 200 randomly selected initial conditions on $\mathbf{C}_{r}$, as a function of $r$ is plotted in Figure 5. The simulation result satisfies the following theoretical estimation:

$$
u_{\max } \approx \sup _{\mid \mathbf{e} \|=10^{-6} 6_{r}}(\|u\|) \leq \sup _{\|\mathbf{e}\|=10^{-6} \sigma_{r}}(\|\mathbf{k}\|\|\mathbf{e}\|)=10^{-6} r\|\mathbf{k}\| \cdot
$$

From the above equation, we see that the param-

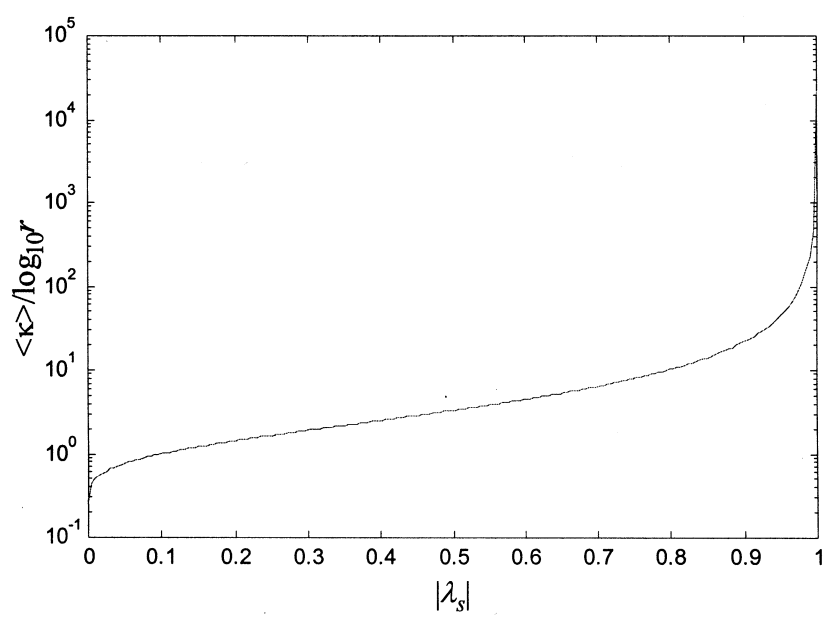

Fig. 4. Normalized average number of iterations $\langle\kappa\rangle / \log _{10} r$ vs. modulus of the stable eigenvalue $\lambda_{s}$ with the OGY method.

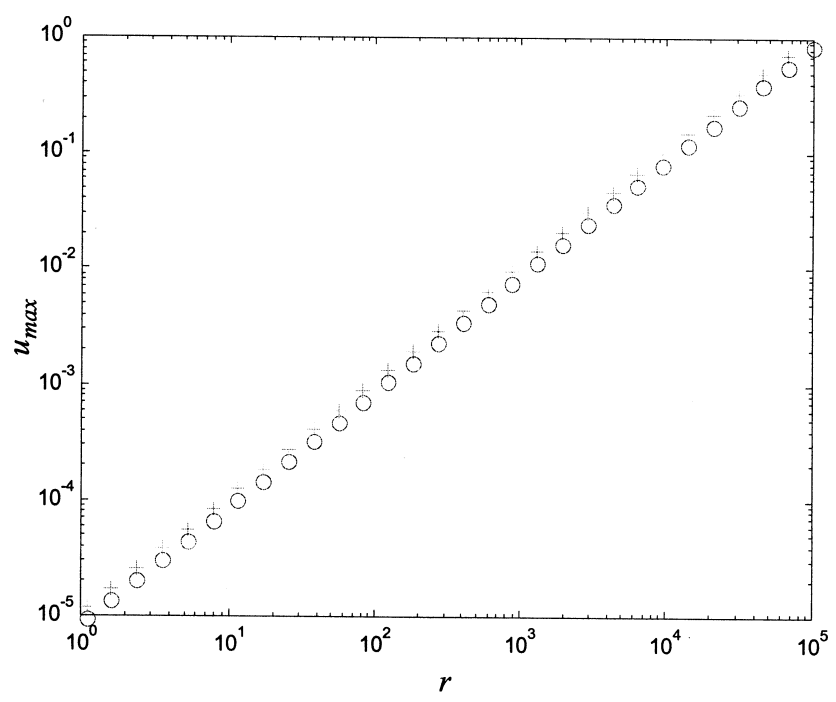

Fig. 5. Maximum input $u_{\max }$ required to reach $\Omega$ from 200 randomly selected initial conditions on $\mathbf{C}_{r}$ vs. parameter $r .(\times)$ the OGY method. ( $\circ$ ) The present method. The result satisfies equation (22).

eter perturbation required for our method is even smaller than that for the OGY method in this example.

\section{CONCLUSIONS}

In this paper, an efficient method for controlling a class of chaotic systems where there is only a single parameter available for adjustment is presented. Since the proposed method is based on a linear approximation of chaotic map, using the embedding technique, it is feasible for practical experiments, where the exact dynamic equations of the chaotic systems are unknown. The proposed method consists of the following procedure. First, one chooses an appropriate unstable periodic orbit embedded in a strange attractor. The linearized model is then estimated in a small defined region around the desired periodic orbit. All of the eigenvalues of the linearized map are then assigned to be zero, using the well-known pole placement technique. According to both theoretical notation and numerical simulations, the proposed method requires only a small parameter perturbation to control chaos, and the convergent properties are superior to the OGY method.

\section{REFERENCES}

1. Moon, F.C., Chaotic Vibrations-An Introduction for Applied Scientists and Engineers, Wiley, New York (1987).

2. Schuster, H.G., Deterministic Chaos, VCH, New York (1988).

3. Thompson, J.M.T. and Bishop, S.R., Nonlinearity and 
Chaos in Engineering Dynamics, Wiley, New York (1994).

4. Ge, Z. M. and Ku, F. N., Stability, "Bifurcation and chaos of a pendulum on a rotating arm," Japanese Journal of Applied Physics, Vol. 40, pp. $7052-7060$ (1997).

5. Ge, Z. M. and Lin, T. N., "Regular and chaotic dynamic analysis and control of chaos of an elliptical pendulum on a vibrating basement," Journal of Sound and Vibration, Vol. 230, pp. 1045-1068 (2000).

6. Chen, C. L. and Yau, H. T., "Chaos in the imbalance response of a flexible Rotor Supported by Oil Film Bearings with Non-Linear Suspension," Nonlinear Dynamics, Vol. 16, pp. 71-90 (1998)

7. Ott, E., Grebogi, C. and York, J.A., "Controlling chaos," Physical Review Letters, Vol. 64, pp. 1196-1199 (1990).

8. Auerbach, D., Grebogi, C., Ott, E. and York, J.A., "Controlling chaos in high dimensional systems," Physical Review Letters, Vol. 69, pp. 3479-3482 (1992).

9. Grebogi, C. and Lai, Y.C., "Controlling chaos in high dimensions," IEEE Trans. Circuits and Systems-I: Fundamental Theory and Applications, Vol. 44, pp. 971-975 (1997).

10. Ushio, T. and Yamamoto, S., "Delayed feedback control with nonlinear estimation in chaotic discrete-time systems," Physics Letters A, Vol. 247, pp. 112-118 (1998).

11. Ushio, T. and Yamamoto, S., "Prediction-based control of chaos," Physics Letters A. Vol. 264, pp. 30-35 (1999).

12. Konishi, K., and Kokame, H., "Observer-based delayedfeedback control for discrete-time chaotic systems," Physics Letters A, Vol. 248, pp. 359-368 (1998).

13. Romeiras, F. J., Grebogi C., Ott, E. and Dayawansa, W. P., "Controlling chaotic dynamical systems," Physica D, Vol. 58, pp. 165-192 (1992).

14. Aston, P. J. and Bird, C. M., "Analysis of the control of chaos-rate of convergence," Int. J. Bifurcation and Chaos, Vol. 5, pp. 1157-1169 (1995)

15. Epureanu, B. I. and Dowell, E. H., "On the optimality of the Ott-Grebogi-Yorke control scheme," Physica D, Vol. 116, pp. 1-7 (1998).

16. Ogata, K., Modern Control Engineering, $2^{\text {nd }}$ Ed., Englewood Cliffs, NJ: Prentice-Hall (1990).

17. Nitsche, G. and Dressler, U., "Controlling chaotic dynamical systems using time delay coordinates," Physica $D$, Vol. 58, pp. 153-164 (1992).

18. Dressler, U. and Nitsche, G., "Controlling chaos using time delay coordinates," Physical Review Letters, Vol. 68, pp. 1-4 (1992) 